\title{
Classification of Atrial Enlargement Using Neural Networks
}

\author{
A. Diery ${ }^{\# 1}$, Y. Abbosh $^{* 2}$, D.V. Thiel ${ }^{\# 3}$, T.R.H. Cutmore ${ }^{\wedge}$, D. Rowlands ${ }^{\# 5}$ \\ ${ }^{\#}$ Griffith School of Engineering, ASchool of Psychology \\ Griffith University, Nathan, Brisbane, Australia, 4111 \\ ${ }^{1}$ adrian.diery@student.griffith.edu.au \\ ${ }^{3}$ d.thielegriffith.edu.au \\ ${ }^{4} t$.cutmorelgriffith.edu. au \\ ${ }^{5} \mathrm{~d}$.rowlandsegriffith.edu. au \\ *College of Electronic Engineering, \\ University of Mosul, Mosul, Iraq. \\ ${ }^{2}$ younisabb@yahoo.com
}

\begin{abstract}
The aim of this study was to classify using a neural network LAE into mildly, moderately, and severely abnormal from a subject's P-wave. Cardiological features, wavelet features, and a combination of both were used to train the neural networks. It was found features derived from the wavelet energy spectrum performed better than the cardiological features on the test cases.
\end{abstract}

\section{INTRODUCTION}

There are several conditions that can cause an enlargement of the atria to occur. Most commonly dilation or hypertrophy of the atrial chambers is due to increased demand upon the cardiac system.[1] This structural change can have significant effects on the overall cardiac function. Assessment of the atrial dimensions can show this change and has been shown to be a predictor of cardiovascular events such as atrial fibrillation and stroke. [2][3][4]. The size of the left atria is commonly used as the indicator for a change in atrial dimensions. The change in the left atrial area can be classified into 3 main groups; mildly abnormal, moderately abnormal, and severely abnormal. [5] The mildly abnormal have left atrial areas of $20-30 \mathrm{~cm}^{2}$, the moderately abnormal have areas of $30-40 \mathrm{~cm}^{2}$, and the severely abnormal have areas $>40 \mathrm{~cm}^{2}$.

The Left atrial enlargement (LAE) has been measured successfully using cardiac ultrasound (echocardiology). The ultrasound is a noninvasive and widely used technique but has the drawback that it is an expensive technique. The electrocardiogram (ECG) is a much cheaper alternative so it is desirable to use it to help identify LAE.

The morphology of the P-wave has been shown to provide insight into the conduction through the atria.[6] It has also been shown that there is a significant correlation between the duration of the widest P-wave in a 12 lead ECG and the long axis dimension in a 2D transthoric echocardiogram (TTE).[7] This indicates that the P-wave can be useful in classifying the degree of Atrial enlargement.

The problem with the P-wave is that it is has a very low signal level and is very noisy which makes it difficult to characterise the morphology. One way that the signal level can be improved is to use signal averaging to improve

978-1-4244-2193-0/08/\$25.00 C2008 IEEE visualisation for manual review by a cardiologist. Previous work by the authors indicate that wavelet analysis of the Pwave can be very sensitive to changes in the morphology and thereby alleviate some of the difficulties inherent in the measurement [8].

Neural networks are a useful technique for pattern matching or feature extraction or classification [6]. Neural networks have been applied to the ECG and in particular the P-wave for pattern matching and feature extraction [6]. This paper proposes to apply neural networks to the P-wave to classify LAE as mildly or moderately or severely enlarged.

\section{Methodology}

The aim of this study was to classify LAE into the 3 groupings of mildly abnormal, moderately abnormal, and severely abnormal from the subject's P-wave using a neural network. Neural networks were used to perform the classification and they were created, trained and tested using 3 sets of ECG features. The first set was the cardiological features since the cardiologist normally examines the P-wave durations and areas to assess the ECG. The second set was the wavelet features since previous studies by the authors [8] have shown that the wavelet analysis of the P-wave was sensitive to changes in morphology. The final set was a combination of both the cardiological and wavelet features. In order to build and test the neural network classifiers the following methodology was used:

1. Extract $1 \mathrm{P}$-wave per subject (beat averaged).

2. Randomly choose 36 subjects for training set with the remainder becoming the testing set ensuring that there were enough subjects from each of the categories.

3. Extract the cardiological features from the training and test data sets.

4. Create and train a neural network based upon the training set of cardiological features.

5. Test the neural network with the test set of cardiological features 
6. Repeat 3-5 using the wavelet features.

7. Repeat 3-5 using both the wavelet and cardiological features.

\section{A. Experimental Data \& P wave Extraction}

Echocardiograms and ECG recordings were taken from 52 subjects during sinus rhythm. The mean age was $76.9 \pm 12.4$ years and there were 37 Males and 15 females. The mix of gender does not affect the result since this study classifies the amount of LAE and does not try to correlate LAE to a particular cause. Each subject had a clinical 12 lead ECG recorded on the same day of their echocardiogram. The ECG recordings were 10 seconds in length and recorded using Micromedical's ECG PC link ${ }^{\mathrm{TM}}$, with a precision of 16 bits at a sampling rate of $1 \mathrm{kHz}$ which is downsampled to $500 \mathrm{~Hz}$ using an averaging and peak picking algorithm for storage. The amplifier input range is $+/-6 \mathrm{mV}$ with a $3 \mathrm{~dB}$ bandwidth of $0.05-175 \mathrm{~Hz}$. All recordings were made with a permanent $1^{\text {st }}$ order high and low pass filter set to $0.05 \mathrm{~Hz}$ and $170 \mathrm{~Hz}$ respectively.

The subjects exhibited echocardiographic characteristics of enlarged left atria with left atrial areas greater than $20 \mathrm{~cm}^{2}$ [5]. One subject had a left atrial area of $19.7 \mathrm{~cm}^{2}$, one subject was a borderline case with a left atrial area of $20 \mathrm{~cm}^{2}$, and the rest were larger than $20 \mathrm{~cm}^{2}$. Overall there was one subject $<20$ $\mathrm{cm}^{2}, 15$ subjects that were mildly abnormal, 33 subjects that were moderately abnormal, and 3 subjects that were severely abnormal. The measurements of left atrial area were made from 4 chamber apical views of 2 dimensional TTE in accordance with clinical procedure recommendations made by the American Society of Echocardiography [9]. The echocardiogram provided a view of the frontal plane of the heart. It is for this reason that it was decided to use the Frank Lead system (X-Y-Z) which can be derived from the 12 lead ECG using the inverse Dower transform [10] [11]. The frontal plane is defined by a horizontal $\mathrm{X}$ axis across the torso and a vertical $\mathrm{Y}$ axis down the torso. It should be noted that $\mathrm{X}$ and $\mathrm{Y}$ directions do not precisely align with the long and short axes of the atria as provided by the TTE.

A beat averaged $\mathrm{P}$ wave signal was produced for each subject from the 10 second clinical ECG recording. The baseline was removed using a $4^{\text {th }}$ order lowpass Butterworth filter with a $3 \mathrm{~dB}$ cutoff at $0.5 \mathrm{~Hz}$. The $\mathrm{P}$-waves were identified by the standard approach involving automated location of the QRS complex as a landmark to search for the P-wave [12] The QRS complexes were isolated by wavelet decomposition and applying a hard threshold to 3 selected scales of wavelet coefficients. The peaks were correlated across all scales to discriminate between QRS complexes and broader high amplitude $\mathrm{T}$ waves.

A search window of $5-25 \mathrm{~ms}$ prior to each QRS complex was processed to identify the $\mathrm{P}$ wave peak. The duration of the $\mathrm{P}$ wave in lead II was derived from this process and served as the standardised measure of the P-wave's duration.

\section{B. Feature Extraction}

1) Cardiological Features: The cardiological parameters are the parameters that can be determined from the durations and areas of the ECG. The parameters chosen are the parameters that cardiologists use to indicate the possibility of atrial enlargement. These parameters are the P-wave duration, the terminal force in V1 (PV1), the P/PR ratio, and the PIIarea.

The $\mathrm{P}$-wave duration is the time in milliseconds between the initial excitation of the sino-atrial node (onset) and the completion of the depolarisation in the left atria (offset). [1315] The P-wave duration is determined from lead II. This is the most common parameter used by cardiologists.

The P-wave terminal force in V1 (PV1) is defined as the product of the duration and amplitude of the P-wave during the negative phase shown in lead V1 [13-15].

The $\mathrm{P} / \mathrm{PR}$ ratio is the ratio of the duration of the $\mathrm{P}$ wave to the duration of the PR segment in lead II [13-15]. The PR segment duration is defined as the time taken from atrial excitation to the onset of ventricular excitation.

The PII area is the area of the P-wave in lead II. It is defined as half the duration multiplied by the maximum amplitude [15].

The cardiological features consisted of 4 values.

$$
\mathrm{V}_{\text {cardio }}=\{\mathrm{P}, \mathrm{PV} 1, \mathrm{P} / \mathrm{PR}, \text { PIIArea }\}
$$

2) Wavelet Features: The Continuous Wavelet Transform (CWT) applied in this study was a continuous scale discrete time transform since the signal being processed is discrete in time. The chosen wavelet for this application was the 2nd order derivative of the Gaussian function. This wavelet was found to provide the highest correlation with the healthy $\mathrm{P}$ wave [8]. The 2 nd order derivative wavelet basis function is defined as:

$\psi(t)=B_{0}\left(1-t^{2}\right) \cdot \exp \left(-\frac{t^{2}}{2}\right)$

The constant, $B_{0}$, is the normalising constant for the wavelet base required to normalise the energy of the basis function. The set of wavelet coefficients and the wavelet energy spectrum were then used to derive the following characterizations of the $\mathrm{P}$ wave:

The total energy contained in each scale (a) of wavelet coefficients $C\{a, b\}$ for a $\mathrm{P}$ wave was defined as:

$\left.E_{v}^{i}(a)=\frac{1}{a^{2}} \sum_{n=p_{1}^{i}}^{p_{2}^{i}} \mid C\{a, b(n))\right\}^{2}$

Where the total energy of the $i_{\text {th }} P$ wave at each level, $E_{\mathrm{v}}{ }^{\mathrm{i}}(\mathrm{a})$, was defined as the sum of all energy at all locations, $\mathrm{b}(\mathrm{n})$, between the onset $p_{1}^{i}$ and offset $p_{2}^{i}$ of the $\mathrm{P}$ wave.

The measure for total energy was defined as the energy 
contained within all scales for the duration of the $\mathrm{P}$ wave. The peak frequency corresponds to the scale which had the highest correlation between the $\mathrm{P}$ wave and the wavelet basis [16].

In deciding on the frequency bands to investigate this study was guided by previous work [8] that found frequencies in the band $13.5-2.5 \mathrm{~Hz}$ to be highly correlated to the overall morphology of the P wave. Frequency bands of coefficients lower than $2.5 \mathrm{~Hz}$ were observed to be significantly affected by adjacent QRS complexes and were therefore discarded from analysis.

Two measures of wavelet parameter stability were used as it has been shown that abnormal $\mathrm{P}$ waves evidence variability [17]. These variance measures were the inter-quartile range and the quartile variation. Both measures describe the regularity and morphology of the P-wave's energy distribution.

The inter-quartile range (IQR) and quartile variation (QV) were defined, respectively as:

$$
\mathrm{IQR}=\mathrm{Q}_{3}-\mathrm{Q}_{1}
$$

$$
\mathrm{QV}=\left(\mathrm{Q}_{3}-\mathrm{Q}_{1}\right) /\left(\mathrm{Q}_{3}+\mathrm{Q}_{1}\right)
$$

The first, $\mathrm{Q}_{1}$, and third, $\mathrm{Q}_{3}$, quartiles are median points between the extremes of each P-wave's energy distribution and its statistical median. The inter-quartile range describes the range of wavelet scales which contain the central $50 \%$ of the total energy of the P-wave. The value of $\mathrm{Q} 2$ gives the statistical median of the P-wave's energy distribution.

The four wavelet parameters derived for each lead were; the peak frequency at the maximum correlation, the IQR, the $\mathrm{QV}$, and Q2. Since the $\mathrm{X}, \mathrm{Y}$ and $\mathrm{Z}$ leads were used then the wavelet features consisted of 12 values.

$$
\begin{gathered}
\mathrm{V}_{\text {wave }}=\left\{X_{\mathrm{pf}}, X_{\mathrm{IQR}}, X_{\mathrm{QV}}, X_{\mathrm{Q} 2,},\right. \\
\mathrm{Y}_{\mathrm{pf}}, \mathrm{Y}_{\mathrm{IQR}}, \mathrm{Y}_{\mathrm{QV}}, \mathrm{Y}_{\mathrm{Q} 2}, \\
\left.\mathrm{Z}_{\mathrm{pf}}, \mathrm{Z}_{\mathrm{IQR}}, \mathrm{Z}_{\mathrm{QV}}, \mathrm{Z}_{\mathrm{Q} 2}\right\}
\end{gathered}
$$

\section{Neural Network Classifier}

Three neural networks have been adopted to classify LAE for abnormal cases. The cases were classified according to their area as mildly, moderately, or severely abnormal [5].

Each suggested neural network had an input layer with a sigmoid transfer function, hidden layers with a linear transfer function, and an output layer with a sigmoid transfer function.

The first neural network took the 4 cardiological features as the input (P, P/PR, PV1, PIIarea). The number of neurons for the three layers were $8-8-3$ respectively.

The second neural network took the 12 wavelet features as the input $\left(\mathrm{X}_{\mathrm{pf}}, \mathrm{X}_{\mathrm{IQR}}, \mathrm{X}_{\mathrm{QV}}, \mathrm{X}_{\mathrm{Q} 2}, \mathrm{Y}_{\mathrm{pf}}, \mathrm{Y}_{\mathrm{IQR}}, \mathrm{Y}_{\mathrm{QV}}, \mathrm{Y}_{\mathrm{Q} 2}, \mathrm{Z}_{\mathrm{pf}}, \mathrm{Z}_{\mathrm{IQR}}\right.$, $\mathrm{Z}_{\mathrm{QV}}, \mathrm{Z}_{\mathrm{Q} 2}$ ). The number of neurons were $20-20-3$ respectively.
The third neural network took the 16 features from the combination of the caridiological and wavelet features. The number of neurons were 20-20-3 respectively.

To train each of the neural networks, 36 subjects were used with the normal back propagation learning method. After training the networks were tested with the 16 other subjects.

\section{RESULTS AND ANALYSIS}

The results for the 16 test cases for each neural network is given in the table below.

TABLE I

CLASSIFICATION RESULTS FOR EACH NEURAL NETWORK

\begin{tabular}{lll}
\hline Input & Correct & Incorrect \\
\hline Cardiological & $11(69 \%)$ & $5(31 \%)$ \\
Wavelet & $13(81 \%)$ & $3(19 \%)$ \\
Both & $13(81 \%)$ & $3(19 \%)$ \\
\hline
\end{tabular}

The results in table 1 indicate that the neural network has better generalisation performance using the wavelet features as inputs. The combination of the ECG features and wavelet features as inputs to the neural network does not significantly improve the classification performance.

The ECG features (PII, PV1) provide parameters of the P wave which are approximations and do not account for variations which can occur, such as alternate directions of conduction. The wavelet features take into account the 3 planes of conduction and provide a less empirical method of parameter extraction. Hence the wavelet features and combinations gave better results than the cardiological features alone. Since the wavelet features and the combination of the wavelet and cardiological features gave the same classification result then it implies that the wavelet features provide more descriptive information to the neural network.

\section{CONCLUSIONS}

The neural network using wavelet features tends to give the better classification results, which indicates that wavelet features are useful inputs for a neural network classifier.

Cardiological parameters assess the shape of the P-wave using generalized methodologies and hence the area measurements are approximations. The accuracy of these features could be improved by an objective automated assessment of the $\mathrm{P}$ wave area.

It should be noted that this is a preliminary study with a limited number of cases with severely dilated atria. So a larger study is required to assess the robustness and sensitivity of this approach.

Overall, this study indicates that a neural network in conjunction with wavelet features derived from clinical ECGs can be used as a classifier for the severity of LAE.

\section{ACKNOWLEDGMENT}

The authors wish to acknowledge the support of Hearts First cardiology clinic in Greenslopes Private Hospital, Brisbane, 
Australia for collecting and providing the ECG and echocardiography data. The authors would especially like to thank Tony Foreshaw and Dr. Roess Pascoe for their assistance.

\section{REFERENCES}

[1] S. Kohsaka, R.R. Sciacca, K. Sugioka, R.L. Sacco, S. Humma, M.R. Di Tullio, "Electrocardiographic Left Atrial Abnormalities and Risk of Ischemic Stroke", Stroke, Vol. 36, pp.2481-2483, Nov 2005.

[2] C.P. Appleton, J.M. Galloway, M.S. Gonzalez, M. Gaballa and M.A. Basnight, "Estimation of left ventricular filling pressures using twodimensional and Doppler echocardiography in adult patients with cardiac disease: additional value of analyzing left atrial size, left atrial ejection fraction and the difference in duration of pulmonary venous and mitral flow velocity at atrial contraction", Journal of the American College of Cardiology, Vol. 22 , pp. 1972-1982, 1993.

[3] A. Rossi, M. Tomaino and G. Golia et al., "Usefulness of left atrial size in predicting postoperative symptomatic improvement in patients with aortic stenosis", American Journal of Cardiology, Vol. 86, pp. 567$570,2000$.

[4] W.P. Abhayaratna, J.B. Seward, C.P. Appleton, P.S. Douglas, J.K. Oh, A.J. Tajik, T.S.M. Tsang, "Left Atrial Size: Physiologic Determinants and Clinical Applications", Journal of the American College of Cardiology, Vol. 47, pp. 2357-2363, 2006.

[5] R.M. Lang, M. Bierig, R. Devereux, F. Flachskampf, E. Foster, P.A Pellikka, M.H. Picard, M.J. Roman, J. Seward, J.S. Shanewise, S.D Solomon, K.T. Spencer, M.StJ. Sutton and W.J. Stewart, "Recommendations for Chamber Quantification: A Report from the American Society of Echocardiography's Guidelines and Standards Committee and the Chamber Quantification Writing Group, developed in Conjunction with the European Association of Echocardiology, a Branch of the European Society of Cardiology", Journal of the American College of Cardiology, Vol. 18, No. 12, pp. 1440-1463, Dec 2005

[6] E. Botter, C.L. Nascimento Jr, and T. Yoneyama, "A Neural Network With Asymmetric Basis Functions for Feature Extraction of ECG P waves", IEEE Trans. on Neural Networks, Vol. 12, No. 5, pp 12521255, Sept 2001.

[7] V. Ariyarajah, L. Prajapat, K.K. Kumar, I. Barac, S. Apiyasawat, D.H Spodick, "Quantitative Estimation of Left Atrial Linear Dimension on a Transthoracic Echocardiogram Using an Electrocardiographic Formulaic Assessment", The American Journal of Cardiology, Vol. 100, Issue 5, pp 894-898, 2007

[8] A. Diery, D. Rowlands, D. James, and T. Cutmore, "Wavelet based analysis of atrial activity", WSEAS Trans. on Sys., Issue 10, Vol. 3, pp 3189-3192, Dec 2004

[9] J. Gottdiener, J. Bednarz, R. Devereux, J. Gardin, A. Klein, W. Manning, A. Morehead, D. Kitzman, J. Oh, M. Quinones, "American Society of Echocardiography recommendations for use of echocardiography in clinical trials. A report from the American society of echocardiography's guidelines and standards committee and the task force on echocardiography in clinical trials.", Journal of the American Society of Echocardiography, Vol. 17, Issue 10, pp 1086-1119.

[10] G.E. Dower, "The ECGD: a derivation of the ECG from VCG leads", Journal of Electrocardiology, April, Vol. 17, Iss. 2, pp. 189-191, 1984.

[11] L. Edenbrandt, O. Pahlm, "Vectorcardiogram synthesized from a 12lead ECG: superiority of the inverse Dower matrix", Journal of Electrocardiology, Vol. 21, Iss. 4, pp. 361-367, 1998.

[12] J. Carlson, R. Johansson, S. B. Olsson, "Classification of Electrocardiographic P-Wave Morphology," IEEE Trans Bio Med Eng., Vol. 48, No. 4, pp. 401-405, April 2001

[13] I.Kasser, J.W. Kennedy, "The relationship of Increased Left Atrial Volume and Pressure to Abnormal P Waves on the Electrocardiogram", Circulation, Vol. 39, pp 339-343, Mar 1969

[14] K.S. Lee, C.P. Appleton, S.J. Lester, T.J. Adam, R.T. Hurst, C.A Moreno, G.T. Altemose, "Relation of Electrocardiographic Criteria for Left Atrial Enlargement to Two-Dimensional Echocardiographic Left Atrial Volume Measurements", American Journal of Cardiology, Vol. 99, pp.113-118, 2007.

[15] J. Birbeck, D.B. Wilson, M.A. Hall, D.G. Myers, "P-wave morphology correlation with left atrial volumes assessed by 2-dimensional echocardiography", Journal of Electrocardiology, Vol. 39, pp 225-229, 2006

[16] O. A. Rosso, "Wavelet entropy: a new tool for analysis of short duration brain electrical signals," Journal of Neuroscience Methods, Vol. 105, pp 65-75, 2001

[17] S. Poli, V. Barbaro, P. Bartolini, G. Calcagnini, F. Censi "Prediction of atrial fibrillation from surface ECG: review of methods and algorithms", Ann Ist Super Sanità, Vol. 39(2), pp.195-203, 2003 